\title{
Early Postnatal Hyperthyroidism Alters Hippocampal Circuitry and Improves Radial-Maze Learning in Adult Mice
}

\author{
H. Schwegler, ${ }^{1}$ W. E. Crusio, ${ }^{2, a}$ H.-P. Lipp, ${ }^{3}$ I. Brust, ${ }^{2}$ and G. G. Mueller ${ }^{1}$ \\ ${ }^{1}$ Anatomisches Institut, Universität Freiburg i.Br., Freiburg, Germany, 2Institut für Humangenetik und Anthropologie, \\ Universität Heidelberg, Heidelberg, Germany, and ${ }^{3}$ Anatomisches Institut, Universität Zürich, Zürich, Switzerland
}

Inbred mice show strain-specific differences in the hippocampal mossy fiber projection. These differences are most pronounced in the portion of the projection that forms synaptic connections with the basal dendrites of the CA3 pyramidal neurons [intra- and infrapyramidal mossy fiber (IIPMF) projection]. We have previously demonstrated that the extent of the IIP-MF subfield is positively correlated with the capacity to perform a spatial radial-maze task and that an experimentally induced enlargement of the IIP-MFs, by means of postnatal thyroxine treatment, predicted the ability of adult two-way avoidance learning. In the present study, we tested whether this treatment would also influence radial-maze performance.

Forty-five male mouse pups from the inbred strain DBA/2 (chosen because of scanty IIP-MF projection and poor radialmaze learning) were divided into three groups that received daily injections of either $2 \mu \mathrm{g}$ L-thyroxine, an alkaline vehicle solution, or physiological saline. Treatment lasted from postnatal days 0 to 11. At the age of 3 months, these animals were tested in an eight-arm radial maze. The extent of their IIP-MF projections was measured by means of planimetry on Timm-stained sections.

Thyroxine-treated animals made significantly fewer errors and had larger IIP-MF projections as compared to both control groups. Within each group, the individual variability of the IIP-MF projection was significantly and positively correlated with performance. We conclude that experimentally modified IIP-MF projections mediate processes underlying spatial working memory. It would appear that the hippocampal circuitry alterations induced by postnatal hyperthyroidism can counteract a hereditary lack of talent, albeit only partially and in selected populations.

Naturally occurring variations of the rodent hippocampal mossy fiber distribution have functional consequences for the regulation of different aspects of behavior. The first indication for such

\footnotetext{
Received Oct. 1, 1990; revised Jan. 28, 1991; accepted Feb. 25, 1991.

We thank Professor M. Frotscher, Professor F. Vogel, and Dr. G. M. Peterson for critically reading the manuscript. H.P.L. was supported by the Swiss National Foundation for Scientific Research Grant 3100-009470 and by the Julius-KlausStiftung für Genetik at the University of Zürich. G.G.M. was supported by a Fulbright stipend.

Correspondence should be addressed to Dr. Herbert Schwegler, Institute of Anatomy, University of Freiburg, Albertstrasse 17, D-7800 Freiburg i.Br., Germany.

a Present address: Groupe Génétique, Neurogénétique et Comportement, U.R.A. 1294 C.N.R.S., U.E.R. Biomédicale, Université Paris V, 45 rue des Saints-Pères, 75270 Paris Cedex 06, France.

Copyright (C) 1991 Society for Neuroscience 0270-6474/91/112102-05\$03.00/0
}

a relationship was a ncgativc correlation between the size of the intra- and infrapyramidal mossy fiber (IIP-MF) projection and two-way active avoidance learning in mice and rats (Schwegler et al., 1981; Schwegler and Lipp, 1983; Lipp et al., 1989). Inbred mouse and rat strains with smaller IIP-MF terminal fields generally showed better avoidance performance than strains with larger ones. Postnatal injections of thyroxine in mice and rats from strains having small IIP-MF projections led to an exuberant growth of infrapyramidal mossy fibers and to poorer avoidance learning (Lipp et al., 1984, 1988). Thus, the hormone treatment showed the opposite effect of lesions of the hippocampal formation, which generally improve shuttle-box performance (O'Keefe and Nadel, 1978). Furthermore, individual differences in the size of IIP-MFs among the control animals remained strongly correlated with avoidance performance. This suggested that the performance variation of the hormone-treated animals was not simply duc to delctcrious cffects of postnatal hyperthyroidism, but to structural alterations within the hippocampal formation.

Two-way active avoidance is a behavior whose underlying mechanisms are poorly understood and still debated (Bolles, 1970; Wilcock and Fulker, 1973; O'Keefe and Nadel, 1978). In contrast, spatial radial-maze tasks appear to be more dependent on hippocampal function, and the leaming processes involved may require more associative capacities than two-way avoidance (Olton et al., 1979). In a series of experiments, we have demonstrated that the ability of mice to master spatial workingmemory tasks in a radial maze is genetically dependent and is strongly correlated with the extent of the IIP-MF projection: the more IIP-MFs, the fewer errors in the learning task (Crusio et al., 1987; Schwegler et al., 1990). Combined with the fact that hippocampal lesions impair learning in such tasks (Olton et al., 1979; Jarrard, 1983) but improve two-way avoidance (Olton and Isaacson, 1968; Antelman and Brown, 1972; O'Keefe and Nadel, 1978), one would expect that a thyroxine-induced hyperplasia of the IIP-MFs in neonatal animals might correct the hereditary deficits in spatial radial-maze learning in mice from strains carrying small IIP-MF projections. To test this hypothesis, postnatal hyperthyroidism was induced in mouse pups from the inbred strain DBA/2 in which we have previously found a relatively modest IIP-MF projection combined with relatively poor spatial working memory in the radial maze (Schwegler et al., 1990), but excellent two-way avoidance learning (Schwegler and Lipp, 1983).

\section{Materials and Methods}

Animals. DBA/2J mice were bred and raised in the animal colony of the Institute of Human Genetics of the University of Heidelberg. The 
Table 1. Means and SEM of hippocampal and behavioral measures

\begin{tabular}{lccr} 
& \multicolumn{3}{l}{ Trcatments } \\
\cline { 2 - 4 } Variable & Thyroxine & Sörensen & NaCl \\
\hline Body weight $(\mathrm{gm})$ & $23.2 \pm 0.7$ & $24.4 \pm 0.4$ & $24.3 \pm 0.4$ \\
Brain weight $(\mathrm{mg})^{* * *}$ & $361 \pm 4$ & $386 \pm 4$ & $390 \pm 3$ \\
Stratum moleculare $\left(\mathrm{mm}^{2} \times 10^{-3}\right)$ & $278 \pm 8$ & $301 \pm 11$ & $282 \pm 9$ \\
Stratum granulosum $\left(\mathrm{mm}^{2} \times 10^{-3}\right)^{* *}$ & $53 \pm 2$ & $63 \pm 2$ & $56 \pm 2$ \\
Regio inferior $\left(\mathrm{mm}^{2} \times 10^{-3}\right)$ & $601 \pm 19$ & $670 \pm 30$ & $649 \pm 24$ \\
\% Stratum oriens & $32.1 \pm 0.4$ & $32.6 \pm 0.4$ & $33.4 \pm 0.5$ \\
\% Stratum pyramidale & $14.4 \pm 0.4$ & $13.7 \pm 0.2$ & $13.8 \pm 0.3$ \\
\% Stratum radiatum & $26.4 \pm 0.3$ & $26.5 \pm 0.2$ & $26.5 \pm 0.4$ \\
\% Stratum lacumesum-moleculare & $5.7+0.2$ & $6.3 \pm 0.2$ & $5.9 \pm 0.2$ \\
\% Hilus (CA4) & $9.4 \pm 0.3$ & $9.9 \pm 0.2$ & $9.2 \pm 0.2$ \\
\% SP-MF* & $12.0 \pm 0.4$ & $11.0 \pm 0.3$ & $11.1 \pm 0.2$ \\
\% IIP-MF** & $1.9 \pm 0.1$ & $1.3 \pm 0.1$ & $1.3 \pm 0.1$ \\
Numbers of errors, day 5*** & $4.3 \pm 0.8$ & $8.7 \pm 1.2$ & $11.3 \pm 1.2$ \\
Running speed, day 5 & $6.6 \pm 0.7$ & $6.3 \pm 0.4$ & $7.6 \pm 0.5$ \\
\hline
\end{tabular}

Significance' levels of ANOVA: ${ }^{*}, p<0.05 ;{ }^{* *}, p<0.01 ;{ }^{* * *}, p<0.001$.

strain has been maintained there for $15 \mathrm{yr}$. All animals were kept under a 12-hr:12-hr dark/light cycle, with lights on at 6 A.M. Food and water were available ad libitum.

Postnatal treatment. Three treatments were applied. Hyperthyroidism was produced by subcutancous injections of a daily dosc of $2 \mu \mathrm{g} \mathrm{L}$-thyroxine dissolved in $0.05 \mathrm{ml}$ of Sörensen's buffer $(\mathrm{pH} \mathrm{9.0)}$, starting at postnatal day 0 and ending at day 11 . Two types of control injections were applied in similar intervals and volumes, one consisting of the Sörensen vehicle solution, the other of physiological saline.

Because hyperthyroidism accelerates development, all pups in a given litter received the same treatment to prevent a competitive advantage of treated over untreated littermates. Litters were obtained from 10 breeding pairs. Because litters were small (two to six pups) and mortality fairly high (up to 50\%), several litters from each pair had to be used. However, subsequent litters from a given pair received different treatments. After weaning, only males were taken and kept together in groups of three or four animals.

Behavior. The radial maze consisted of a central platform and eight arms made of transparent Plexiglas. The central part measured $22 \mathrm{~cm}$ in diameter. The closed arms were $25 \mathrm{~cm}$ long, $6 \mathrm{~cm}$ high, and $6 \mathrm{~cm}$ wide. Several fresh food pellets were deposited beyond the end of each arm behind a perforated wall. This served to prevent the animals from selecting a baited arm by smelling the presence or absence of a reward. All arms were baited by placing a food pellet (about $10 \mathrm{mg}$ ) behind a low barrier. The maze was placed on the floor, and several extramaze cues were provided close to the maze and between the arms. At the start of each trial, the mouse was placed in the center of the maze and allowed free choice of all eight arms. Between choices the mouse was confined in the center of the maze for $5 \mathrm{sec}$ by means of transparent guillotine doors at the entrance of each arm.

Mice received a $10-\mathrm{min}$ habituation trial with free access to all arms $24 \mathrm{hr}$ prior to training. Subsequently, they were deprived of food but not of water. During training, the body weight was kept at about $85 \%$ of the pretest body weight. Training took place on five consecutive days with one trial per day. A trial was terminated when the animal had eaten all rewards or after $15 \mathrm{~min}$. Entry to an arm was counted when the mouse had entered with all four paws. An error was noted if an animal entered an arm previously visited, or if it did not eat the pellet. The number of errors and running speed in $\mathrm{cm} / \mathrm{sec}$ was recorded for each day of training. Only data from the last $3 \mathrm{~d}$ of training were used for subsequent analysis. For calculating morphobehavioral correlations, only behavioral data from day 5 of training were used. This variable was chosen because in previous experiments it had revealed the clearest strain differences (Schwegler et al., 1990). Because of the obvious effects of thyroxine (smaller size of the animal, higher reactivity), a blind behavioral analysis was not possible.

Histology and morphometry. After training, all brains of the mice were processed for Timm's staining (for details, see Schwegler and Lipp, 1983). Brains were weighed after perfusion with a $1.17 \%$ solution of phosphate-buffered sodium sulfide ( $\mathrm{pH} 7.4$ ) for $1 \mathrm{~min}$, followed sequentially by a phosphate-buffered solution of $3 \%$ glutaraldehyde for 5 min and a 5 -min rinse with the sodium sulfide solution. The brains were postfixed overnight in a buffered sucrose solution containing $3 \%$ glutaraldehyde and then cut in $40-\mu \mathrm{m}$ horizontal sections using a cryostat. Sections were mounted on glass slides, developed for 45-60 min in Timm's solution containing a protective colloid, hydroquinone, citric acid, and silver nitrate, and finally embedded in Merckoglass.

For morphometry, 10 sections per animal were sampled starting immediately below the ventralmost part of the septal pole of the hippocampus. Taking every other one, five sections either from the right or left hippocampus were analyzed per animal.

Morphometric analysis was carried out by measuring of outlines and distributions of Timm-stained bands and patches on drawings made with a projection microscope (magnification, $160 \times$ ) on a graphics tablet connected to a desk computer (for details, see Schwegler and Lipp, 1983). The area covered by the scattered IIP-MF projection was obtained by overlaying the drawings with a point grid and counting the number of points coincident with the Timm reaction product.

The hippocampal variables used for the correlative study were the mean absolute sizes of the strata moleculare and granulosum of the fascia dentata, $\mathrm{CA} 3 / \mathrm{CA} 4$, and the mean relative sizes (expressed as percentage of $\mathrm{CA} 3 / \mathrm{CA} 4$ ) of the following hippocampal subfields: strata oriens, pyramidale, lucidum [suprapyramidal mossy fibers (SP-MFs)], radiatum, and lacunosum-moleculare and the intra- and infrapyramidal mossy fiber (IIP-MF) projection. The morphological analysis was carried out blindly.

Statistics. Treatment effects were assessed by means of a nonparametric analysis of variance (Kruskal-Wallis test; Siegel, 1956). Spearman's rank correlation $\left(r_{\mathrm{s}}\right)$ was used to estimate correlation coefficients.

\section{Results}

\section{Behavior}

Thyroxine-treated mice made fewer errors in the radial-maze task than either saline or Sörensen-treated controls (Table 1). This difference was highly significant at the fifth day of training, as revealed by a Kruskal-Wallis one-way ANOVA $(H=22.11$; $\mathrm{df}=2 ; p<0.001)$. It could be observed also on day 3 of training and marginally on day 4 . Although the hormone-treated mice showed enhanced reactivity as indicated by increased jumping and escapes during handling, their running speed did not differ from that of the controls.

\section{Morphology}

Postnatal thyroxine injections resulted in significantly decreased brain weight $(H=18.20 ; \mathrm{df}=2 ; p<0.001)$, but not body weight (Table 1), and in an enlargement of the relative area of the IIP. MF projection by approximately $45 \%$ (Table 1, Fig. 1). No 

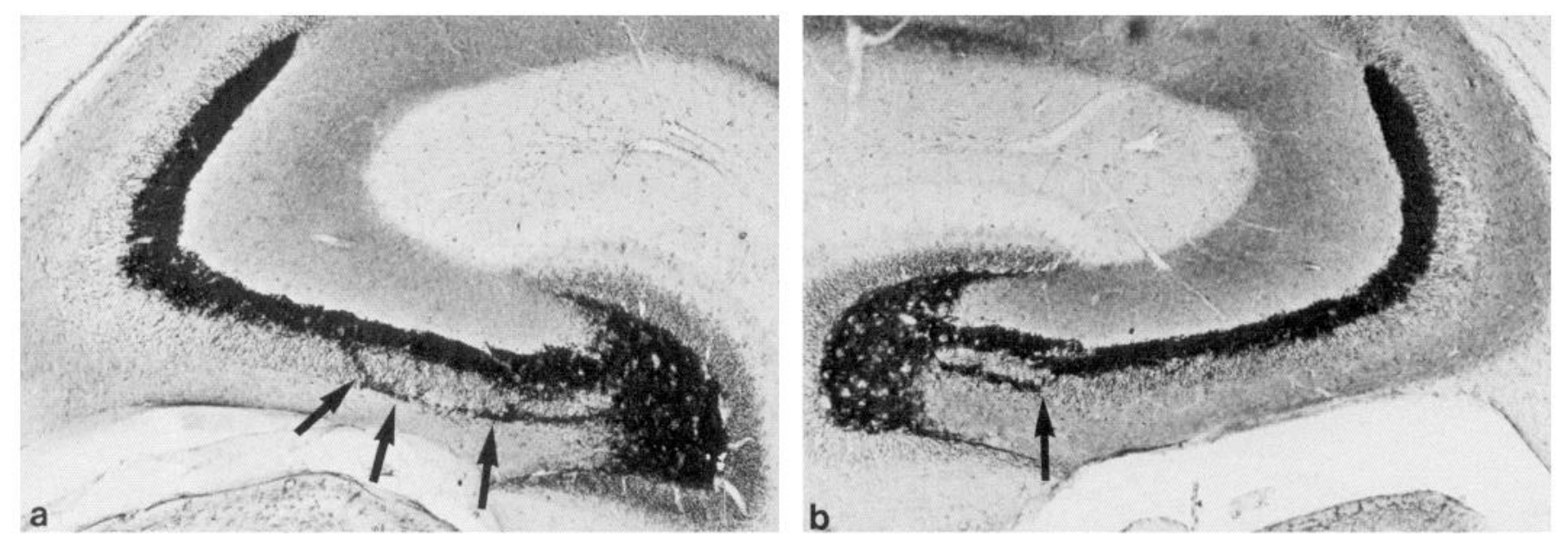

Figure 1. a, Timm-stained hippocampus of an adult DBA/2 mouse in which postnatal hyperthyroidism induced a marked hyperplasia of the intra- and infrapyramidal mossy fibers (indicated by arrows). $b$, Same region of an adult DBA/ 2 control mouse treated with Sörensen's buffer alone. Note the markedly smaller size of the IIP-MF (arrow).

differences were found between the two control groups. The proliferation of the IIP-MFs was most pronounced in the hilar parts of CA3. However, in a few animals, a small band of IIPMFs was observed near the border of CA2. In most mouse strains, Timm reactivity appears only sparsely scattered in this location. Among the other hippocampal subfields, the relative size of the SP-MF projection was about $8 \%$ larger in thyroxinetreated mice (Table 1). In the fascia dentata, the Sörensen-treated group had a larger granule cell layer as compared to the other groups. The size of the molecular layer did not differ between the groups. Otherwise, the morphology of the Timm-stained hippocampi did not show aberrations as compared to untreated $\mathrm{DBA} / 2$ mice.

\section{Morphobehavioral correlations}

The within-group variability of the IIP-MF projection was about equal in the treatment groups. However, within each group, the error scores on day 5 were still negatively and significantly correlated with the IIP-MFs (Fig. 2), most strongly in the thyroxineand Sörensen-treated animals. In the thyroxine-treated group, the behavioral score was also correlated with the size of the stratum oriens $\left(r_{\mathrm{S}}=0.57 ; \mathrm{df}=13 ; p<0.05\right)$ and with the SPMF projection $\left(r_{\mathrm{s}}=-0.61 ; \mathrm{df}=13 ; p<0.05\right)$. In the Sörensentreated control group, an additional correlation occurred only with the relative size of the stratum lacunosum-moleculare $\left(r_{\mathrm{s}}\right.$ $=0.58 ; \mathrm{df}=13 ; p<0.05)$. The occurrence of such within-group correlations shows that the between-group differences in behavior could hardly have been due to unconsciously biased recording of behavior (see Materials and Methods).

\section{Discussion}

The results of this study show that variations of the IIP-MF projections are positively correlated with performance in a spatial radial-maze task as it has been predicted by our former data (Lipp et al., 1988). They are in agreement with results of hippocampal lesion studies in rats that generally have revealed a substantial impairment of spatial radial-maze learning (Olton et al., 1979; Jarrard, 1983). The origin of the structural variations appears to be irrelevant for the observed correlation, because it was found in both the thyroxine-treated and the control animals. This bears a remarkable similarity to the results of the previous study on thyroxine-induced variation of IIP-MFs and two-way avoidance learning (Lipp et al., 1988). The morphometrical results from both studies are largely similar, the observed hyperplasia of the IIP-MFs being slightly less in the present study. This probably reflects minor methodological differences in the quantitative analysis between different experimentators (cf. Schwegler and Lipp, 1983). In the previous study, it was found that the within-group variability of the IIP-MFs in 75 treated DBA/2 mice covaried strongly yet negatively with two-way avoidance performance (Lipp et al., 1988). Precisely the same phenomenon was found in the present study in another 45 animals, with the exception of a different sign of the correlations, this reversal being predicted from lesion studies of the hippocampus and from between-strain correlations. Thus, the observed correlation between hippocampal mossy fiber variations and two entirely different but hippocampus-dependent behaviors strongly suggests that mossy fiber variations are, indeed, influencing hippocampal processing. The results of the two-way avoidance study had also led to the suspicion that the decrease in avoidance learning associated with larger projections did not reflect an impairment, but rather an improvement of hippocampal function. This hypothesis appears to be corroborated by the present results.

Taken together, the data presented here as well as our earlier data provide evidence that variations in the extent of the IIPMF projection, or of an intrahippocampally associated variable thereof, determine one of the basic parameters of hippocampal physiology that is capable of influencing radial-maze learning in a linear fashion. The nature of the physiological process associated with size variations of the IIP-MF projection is unknown. Recent data suggest that it may relate to long-term potentiation (LTP), because slice preparations from mouse strains with differential mossy fiber projections have shown a larger population spike amplitude after tetanic stimulation in mouse hippocampi with extended IIP-MF projections (Heimrich et al., 1989). One may notice here also that LTP involving mossy fiber synapses is distinctly different from LTP occurring in associational synapses of the Schaffer collaterals (Zalutsky and Nicoll, 1990), suggesting functional specialization of hippocampal projection systems. Equally possible appears an association between extent of the IIP-MF projection and hippocampal activity levels 

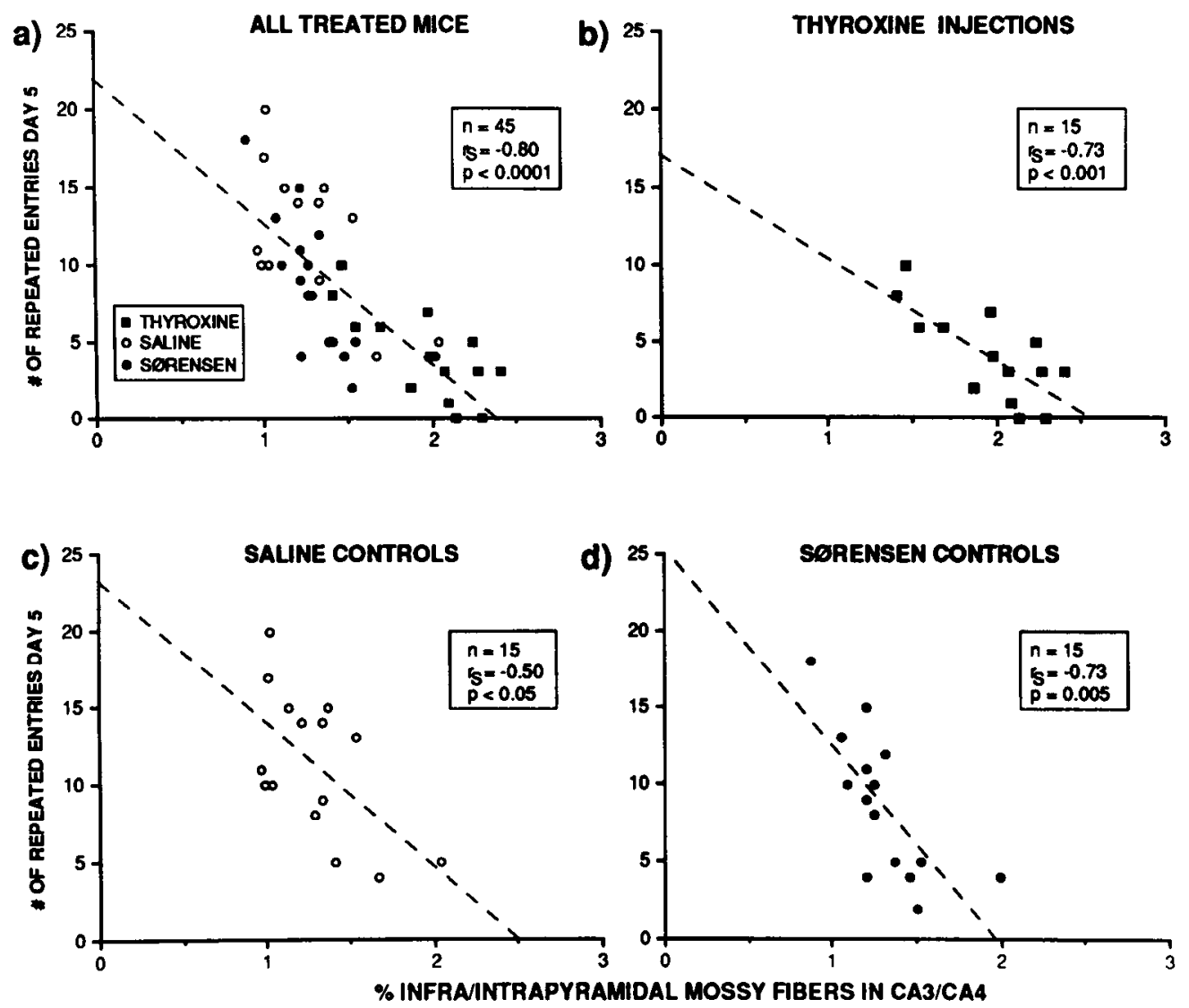

Figure 2. Scatter plots of the relationship between the number of errors on day 5 and the size of the IIP-MF terminal field in different experimental groups. The significance levels are onetailed, because the direction of the correlation has been predicted.

of protein kinase $\mathrm{C}$, which have been shown to correlate with swimming navigation learning in mice (Wehner et al., 1990). This unknown process might also reflect a functional balance between lamellar (mossy fibers) and associational input (Schaffer collaterals). In this case, one would expect that an increased lamellar input decreases the impact of remote hippocampal regions, keeping the local activity levels resistant towards extraneous influences. Perhaps such local functional stability is necessary for the formation of memory.

Apart from the changes in the IIP-MF projection, the postnatal hyperthyroidism had some visible morphological side effects, such as reduced brain weight. The relatively high, and presumably somewhat toxic, dosage was chosen because earlier studies had shown that predictable alterations of the IIP-MF projections could not be obtained reliably using lower doses. Nevertheless, the observed correlations between IIP-MFs and radial-maze performance are probably not due to side effects of thyroxine. This is suggested by the clear within-group correlations in the controls. One must notice, however, that postnatal hyperthyroidism has been found to impair maze learning and intellectual performance (Eayrs, 1964; Davenport and Gonzalez, 1973; Stone and Greenough, 1975; Daneman and Howard, 1980). In vicw of these findings, we emphasize that the astonishing observation of beneficial effects of strong hyperthyroidism has only been demonstrated in particular rodent populations characterized by hereditary scanty IIP-MF projections.

Our results lead to the following conclusions: First, the strong effects of early postnatal thyroxine treatment on the IIP-MFs counteract the genetically determined defect of DBA/2 mice in mastering the present spatial working-memory task. Second, this finding seems to be quite robust, because the thyroxine- independent variation induced in the inbred control group also correlates with learning in such a way that animals with larger IIP-MF projections make fewer errors. This is in agreement with our earlier findings that genetically dependent variation of the IIP-MFs also correlates positively with spatial radial-maze learning (Schwegler et al., 1990). Third, behavior covaries with the anatomical trait independent of the origin of the variation in this trait. Fourth, this relationship is strong enough to overcome other effects of hyperthyroidism that might have negative effects on learning performance.

It has now been shown twice, in vastly different test paradigms (shuttle box and radial maze), that behavioral differences of adult mice follow the structural variations of the IIP-MFs rather precisely, regardless of the source of these variations. We conclude that these anatomico-behavioral correlations reflect a functional involvement of the IIP-MFs in the regulation of learning and memory in mice.

\section{References}

Antelman SM, Brown TS (1972) Hippocampal lesions and shuttlebox avoidance behavior: a fear hypothesis. Physiol Behav 9:15-20.

Bolles RC (1970) Species-specific defense reactions and avoidance learning. Psychol Rev 77:32-48.

Crusio WE, Schwegler H, Lipp H-P (1987) Radial-maze performance and structural variation of the hippocampus in mice: a corrclation with mossy fibre distribution. Brain Res 425:182-185.

Daneman D, Howard NJ (1980) Neonatal thyrotoxicosis: intellectual impairment and craniosynostosis in later years. J Pediatr 97:257259.

Davenport JW, Gonzalez LM (1973) Neonatal thyroxine stimulation in rats: accelerated maturation and subsequent learning deficit. J Comp Physiol Psychol 85:397-408. 
Eayrs JT (1964) Effect of neonatal hyperthyroidism on maturation and learning in the rat. Anim Behav 12:195-199.

Heimrich B, Claus H, Schwegler H, Haas HL (1989) Hippocampal mossy fiber distribution and long-term potentiation in two inbred mouse strains. Brain Res 490:404-406.

Jarrard LE (1983) Selective hippocampal lesions and behavior: effects of kainic acid lesions on performance of place and cue tasks. Behav Neurosci 97:873-889.

Lipp H-P, Schwegler H, Driscoll P (1984) Postnatal modification of hippocampal circuitry alters avoidance learning in adult rats. Science 225:80-82.

Lipp H-P, Schwegler H, Heimrich B, Driscoll P (1988) Infrapyramidal mossy fibers and two-way avoidance learning: developmental modification of hippocampal circuitry and adult behavior of rats and mice. J Neurosci 8:1905-1921.

Lipp H-P, Schwegler H, Crusio WE, Wolfer DP, Heimrich B, Driscoll $P$, Leisinger-Trigona M-C (1989) Using genetically-defined rodent strains for the identification of hippocampal traits relevant for twoway avoidance learning: a non-invasive approach. Experientia 45: 845-859.

O'Keefe J, Nadel L (1978) The hippocampus as a cognitive map. Oxford: Clarendon.

Olton DS, Isaacson RL (1968) Hippocampal lesions and active avoidance. Physiol Behav 3:719-724.

Olton DS, Becker JT, Handelmann GE (1979) Hippocampus, space, and memory. Behav Brain Sci 2:313-365.
Schwegler H, Lipp H-P (1983) Hereditary covariations of neuronal circuitry and behavior: correlations between the proportions of hippocampal synaptic fields in the regio inferior and two-way avoidance in mice and rats. Behav Brain Res 7:1-39.

Schwegler H, Lipp H-P, Van der Loos H, Buselmaier W (1981) Individual hippocampal mossy fiber distribution in mice correlates with two-way avoidance performance. Science 214:817-819.

Schwegler H, Crusio WE, Brust I (1990) Hippocampal mossy fibers and radial-maze learning in the mouse: a correlation with spatial working memory but not with non-spatial reference memory. Neuroscience 34:293-298.

Siegel S (1956) Nonparametric statistics for the behavioral sciences. New York: McGraw-Hill.

Stone JM, Greenough WT (1975) Excess neonatal thyroxine: effects on learning in infant and adolescent rats. Dev Psychobiol 8:479-488.

Wehner JM, Sleight S, Upchurch M (1990) Hippocampal protein kinase $\mathrm{C}$ activity is reduced in poor spatial learners. Brain Res 523: 181-187.

Wilcock J, Fulker DW (1973) Avoidance learning in rats: genetic evidence for two distinct behavioral processes in the shuttle box. $J$ Comp Physiol Psychol 82:247-253.

Zalutsky RA, Nicoll RA (1990) Comparison of two forms of longterm potentiation in single hippocampal neurons. Science 248:16191624. 